\title{
Early childhood CMV infection may decelerate the progression to clinical type 1
}

\section{diabetes}

Ilse Ekman ${ }^{1,2}$, Tytti Vuorinen ${ }^{3}$, Mikael Knip ${ }^{4,5,6,7}$, Riitta Veijola ${ }^{8}$, Jorma Toppari ${ }^{9,10}$, Heikki Hyöty ${ }^{11,12}$, Tuure Kinnunen ${ }^{2,13}$, Jorma llonen ${ }^{1,14}$, Johanna Lempainen ${ }^{1,10}$

\section{Running title: Role of cytomegalovirus infection in T1D}

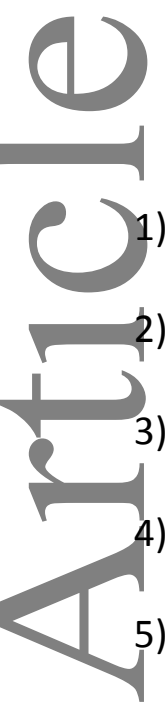

Immunogenetics laboratory, Institute of Biomedicine, University of Turku, Turku, Finland

2) Department of Clinical Microbiology, University of Eastern Finland, Kuopio, Finland

3) Department of Virology, University of Turku and Turku University Hospital, Turku, Finland

4) Children's Hospital, University of Helsinki and Helsinki University Hospital, Helsinki, Finland

5) Research Programs Unit, Diabetes and Obesity, University of Helsinki, Finland

6) Center for Child Health Research, Tampere University Hospital, Tampere, Finland

7) Folkhälsan Research Center, Helsinki, Finland

8) Department of Pediatrics, Medical Research Center, PEDEGO Research Unit, University of Oulu and Oulu University Hospital, Oulu, Finland

9) Institute of Biomedicine, University of Turku

10) Department of Pediatrics, University of Turku and Turku University hospital, Turku, Finland

11) Department of Virology, University of Tampere, Tampere, Finland

12) Fimlab Laboratories, Pirkanmaa Hospital District, Tampere, Finland

13) Eastern Finland Laboratory Centre (ISLAB), Kuopio, Finland

14) Clinical Microbiology, Turku University Hospital, Turku, Finland

Corresponding author:

This article has been accepted for publication and undergone full peer review but has not been through the copyediting, typesetting, pagination and proofreading process, which may lead to differences between this version and the Version of Record. Please cite this article as doi: $10.1111 /$ pedi.12788 
Johanna Lempainen, MD, PhD

Immunogenetics Laboratory, Medicity Research Laboratories,

University of Turku, Tykistökatu 6A, FI-20520 Turku, Finland

Tel: +358407665976

E-mail: johanna.lempainen@utu.fi

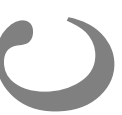

\section{Abstract}

$(1)$

\section{Aims/Hypothesis}

Evidence of the role of cytomegalovirus (CMV) infection in the pathogenesis of type 1 diabetes

(T1D) has remained inconclusive. Our aim was to elucidate the possible role of CMV infection in the initiation of islet autoimmunity and in the progression to clinical T1D among children with HLA-conferred T1D risk.

\section{Methods}

(2)

A total of 1402 children from the prospective Type 1 Diabetes Prediction and Prevention (DIPP) study were analyzed for CMV-specific IgG antibodies during early childhood. All children carried HLA-DQ genotypes associated with increased risk for T1D. The effect of CMV infection on the appearance of T1D-associated autoantibodies (IAA, GADA and IA-2A, n=356) and on the progression rate to clinical T1D ( $n=233$ ) were analyzed with Kaplan-Meier survival analysis and logrank test.

Results 
Early childhood CMV infection was inversely associated with the development of T1D during childhood. Cumulative progression to T1D was decreased in subjects with an early CMV infection $(p=0.035)$. In further analyses, the effect of early CMV infection on the initiation of islet autoimmunity and progression to clinical T1D were examined separately. Interestingly, early CMV infection did not affect the appearance of T1D-associated autoantibodies but a decelerating effect was observed on the progression rate from islet autoimmunity to clinical T1D $(p=0.015)$.

\section{Conclusion}

1

1

Our results suggest that an early childhood CMV infection may decelerate the progression from islet autoimmunity to clinical T1D among at-risk children and may thus protect these children from progressing to T1D during childhood.

Keywords: cytomegalovirus, CMV, type 1 diabetes, autoantibodies, 


\section{Introduction}

Type 1 diabetes (T1D) is considered to arise from complex gene-environmental interactions that affect the immune system. From the heterogeneous group of environmental factors implicated, viruses have been a major target of interest. Several viruses, including enteroviruses and cytomegalovirus (CMV) have been suggested to affect the pathogenesis of T1D. However, the

results of previous studies concerning the role of most viruses, including CMV, have been largely controversial [1].

1

CMV is a common herpes group virus that causes a lifelong latent infection. CMV seroprevalence in adults varies geographically from $40 \%$ to $100 \%$ [2]. In Finland, similar to other Western countries, approximately $50 \%$ of pregnant women are seropositive for CMV-specific IgG antibodies [3]. More than $75 \%$ of childhood CMV infections are acquired in early childhood, transmitted typically perinatally or during the first year of life through saliva, maternal genital secretions or breast milk after reactivation of the latent virus in the mother. Thereafter, the incidence rate of CMV infection, stays rather stable until it peaks again at the age of 4 to 5 years. $[4]$.

We have earlier analyzed the association of perinatal CMV infection and the appearance of early signs of islet autoimmunity and clinical T1D in a prospective cohort of children with HLA-conferred susceptibility to T1D [5]. In that analysis, no association of perinatal CMV infection with islet autoimmunity was observed. In contrast, a trend for a protective effect of perinatal CMV infection on the development of clinical T1D was seen, but the result was statistically non-significant. In the current study, we aimed to further clarify the role of perinatal CMV infection in the pathogenesis of T1D by analyzing samples from a larger cohort of children with HLA-associated T1D risk. 


\section{Methods}

\section{Study cohort}

The study subjects participated in the prospective Finnish Type 1 Diabetes Prediction and Prevention (DIPP) study. In the DIPP study, children born at three university hospitals (Turku, Tampere and Oulu) are first screened for HLA-DR/DQ associated susceptibility to T1D [6]. Children carrying selected HLA genotypes associated with increased T1D risk are recruited to a follow-up program that includes regular blood sampling for measurement of islet autoantibodies. In Turku, samples are collected at 3-month intervals until the age of 24 months, and thereafter at 6-month intervals. In Oulu and Tampere, samples are collected at the ages of 3, 6, 12, 18 and 24 months, and thereafter at yearly intervals. Before 2003 , the appearance of classical islet cell autoantibodies (ICA) was tested using an immunofluorescence method, and if positive, insulin autoantibodies (IAA), antibodies to the $65 \mathrm{kD}$ isoform of glutamic acid decarboxylase (GADA) and antibodies to the insulinoma antigen-2 (IA-2A) were subsequently tested from all samples of these subjects [7]. Since 2003, these three biochemical autoantibodies have been tested from all samples. After the appearance of autoantibodies, the sample collection frequency is raised to every three months in all of the study centers.

The study cohort comprised 356 children who tested persistently positive, i.e. in two consecutive positive samples taken 3 months apart, for at least one of the biochemical islet autoantibodies (IAA, GADA, IA-2A). 1046 autoantibody-negative control subjects matched for age, gender and

clinical center were used as controls. Among the autoantibody-positive subjects, 233 children developed clinical T1D during the follow-up. All subjects were analyzed for CMV-specific IgGantibodies from serum samples obtained between the age of 9 and 24 months. Sampling was conducted independently from the case status with the serum samples available. The length of the 
prospective follow-up for the appearance of islet autoantibodies ranged from 0.9 to 16.0 years (median 11.9 years) measured from birth to the age of seroconversion or the age at the end of the follow-up. For clinical T1D, the length of follow-up ranged from 0.8 to 16.0 years (median 14.8 years) measured from birth to the age of diagnosis or the age at the end of the follow-up. The children were followed until the age of 16 years.

Diabetes was diagnosed according to the WHO criteria. The study protocol was approved by the local ethics committees and a written informed consent was obtained from all parents of the children participating in the study.

\section{Study design}

The effect of perinatal CMV infection on the development of clinical T1D, and separately, on the appearance of islet autoantibodies, and on the progression rate from the initiation of islet autoimmunity, i.e. appearance of islet autoantibodies, to clinical T1D was analyzed.

\section{Cytomegalovirus IgG-antibody measurement}

CMV-specific IgG antibodies were determined from serum samples using an in-house enzyme immune assay described in detail earlier [8]. The cut-off value for positivity was set to $\geq 0.15$ OD.

To improve accuracy, samples with absorbance readings slightly below the reliable cut-off value (0.11-0.149 OD) were excluded $(n=41)$ from the final statistical analyses.

\section{Statistical analysis}

Statistical analyses were performed with SPSS statistics 23. Kaplan-Meier survival analysis with Log Rank test was applied to compare the effect of early CMV infection on the development of T1D, on the appearance of islet autoantibodies, and on the progression rate from the initiation of islet 
autoimmunity to clinical T1D. Chi-square test was used to compare the frequencies of clinical T1D in children positive or negative for CMV-specific IgG antibodies.

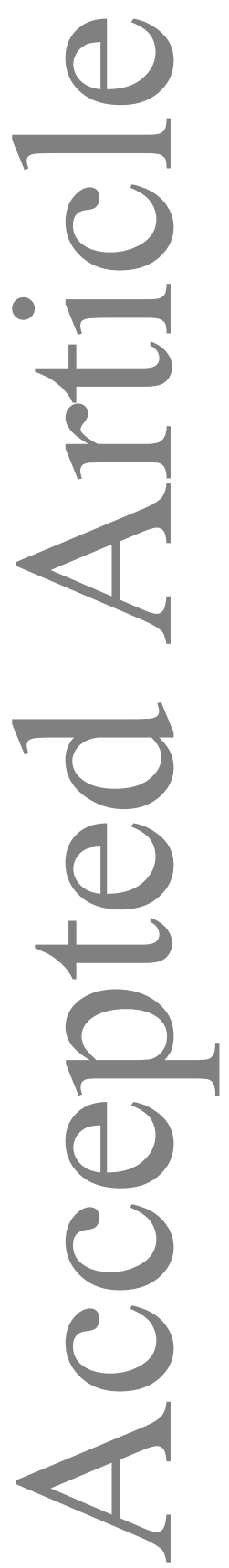




\section{Results}

The prevalence of an early CMV infection was determined by measuring CMV-specific IgG antibodies before the age of 2 years in 1361 (97.1\%) of the 1402 children studied from the DIPP follow-up cohort and these were included in the statistical analyses. From these children, 321 (23.6\%) tested positive for CMV antibodies indicating an early, most probably perinatal, CMV infection (Tables 1).

(

Progression of children with an early CMV infection to clinical T1D during the follow-up was

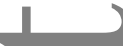
significantly decreased compared to the children without an early CMV infection $(p=0.035$, KaplanMeier survival analysis, log-rank test) (Figure 1). The number of children developing T1D in the group with an early CMV infection was $42 / 321$ (13.1\%) compared to $186 / 1040(17.9 \%)$ in those

\footnotetext{
without $C M V$ infection ( $P=0.044$, Chi-square test $)$.

To further investigate the role of early CMV infection in the pathogenesis of T1D, we analyzed separately the effect of CMV infection on the initiation of islet autoimmunity characterized by the appearance of islet autoantibodies, and on the progression rate of established islet autoimmunity to clinical T1D (Table 2.). No significant effect of early CMV infection was observed on the appearance of islet autoantibodies ( $p=0.357$, Kaplan-Meier survival analysis). Among the group of 321 children with an early CMV infection, 76 (23.7\%) developed humoral signs of beta-cell autoimmunity compared to 269 (25.9\%) among the 1040 children without CMV infection in infancy (Figure 2).
}

As there was no effect between early childhood CMV infection and the development of islet autoantibodies, we set out to explore whether the decreased progression to clinical T1D would be attributable to a reduced rate of progression from the initiation of autoimmunity to clinical T1D. Indeed, we observed that the progression rate was decelerated among autoantibody-positive 
children with an early CMV infection compared to children without CMV infection during infancy ( $p=0.015$, Kaplan-Meier survival analysis) (Figure 3). During the follow-up, 41 (53.9\%) autoantibody-positive children with early CMV infection progressed to clinical T1D compared to 181 (67.3\%) autoantibody-positive children without CMV infection during infancy $(P=0.032$, Chi square test).

In further analyses, no significant effect of early CMV infection on the appearance of a second or third autoantibody was detected: $n=59(18.4 \%)$ vs $n=221(21.3 \%)$ for the appearance of a second autoantibody ( $p=0.207$, Kaplan-Meier survival analysis) and $n=34(10.6 \%)$ vs $n=138(13.3 \%)$, for the appearance of third autoantibody $(p=0.107)$, in CMV-seropositive and -seronegative groups, respectively. We neither observed any difference between the groups in the progression rate to clinical T1D from the appearance of the second or third persistent islet autoantibody: $n=38$ (64.4\%) vs $n=162(73.3 \%)$ for progression to T1D after the appearance of the second islet autoantibody $(p=0.224)$ and $n=25(73.5 \%)$ vs $n=110(79.7 \%)$ for progression to T1D after the appearance of the third islet autoantibody $(p=0.222)$ in CMV-seropositive and -seronegative groups, respectively.

We also tested whether CMV infection had an effect on the appearance of the islet autoantibodies (IAA, GADA or IA-2A) separately. Early CMV infection did not affect the appearance of GADA or IA$2 \mathrm{~A}(\mathrm{p}=0.652$ and 0.224 , respectively). However, a tendency for a protective effect on the appearance of IAA was observed $(p=0.072)$. 


\section{Discussion}

Several virus infections have been implicated in the pathogenesis of T1D. Accumulating data support an association between enterovirus infections and T1D, but the role of other virus infections has remained unclear. Currently, there is no definite evidence for a role of CMV infection in the pathogenesis of T1D. [1].

In Western countries CMV infection is typically acquired during the perinatal period and infancy but less commonly during later childhood [4]. Early childhood is also a critical time period for the 1

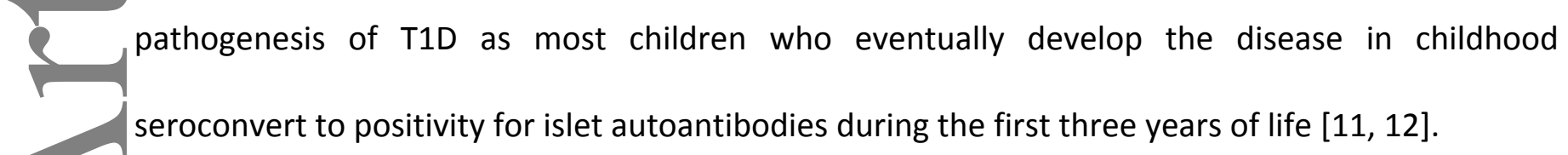

We have earlier analyzed the possible effect of early, mostly perinatal CMV infection on development of T1D in a smaller cohort of children with increased genetic risk for the disease [5]. That study did not suggest an increased risk associated with early CMV infection but instead demonstrated a trend for a decreased risk of T1D among subjects with CMV infection. In the current study, we aimed to further assess the possible effect of early-life CMV infection by analyzing an extended cohort of children participating in the DIPP study. Here, we observed a statistically significant protective effect of an early CMV infection for the development of T1D that was due to a decelerated progression rate to T1D in subjects who had already established islet autoimmunity. Our observations suggest that early-life CMV infection delays further progression of an already established autoimmune process but does not directly prevent the onset of early islet autoimmunity. In accordance with our findings, observation of a protective role of CMV infection in the pathogenesis of another autoimmune disease, celiac disease, has been reported [13]. This finding was also recently confirmed in a large population-based analysis comparing the 
prevalence of antibody-positivity to CMV and other herpes group viruses and positivity for celiac disease-associated transglutaminase type 2 antibody (TG2A) among children [14].

CMV infection is known to have a strong immunomodulatory effect. For example, CMV infection increases the number of effector memory $T$ cells, influencing the composition of the adaptive immune cell repertoire and has been shown to cause an age-dependent decline of the diversity of

the T-cell pool. $[15,16]$. According to our recent unpublished results analyzing samples from the DIPP cohort, perinatal CMV infection induces alterations in the CD8+ memory and CD8+ naïve Tcell compartments, and to a lesser extent in the CD4+ T-cell compartments. These effects last at least until the age of 2 years (Öling $V$ et al., unpublished observations), indicating a strong and long-lasting effect of perinatal CMV infections on the developing immune system.

Perinatal infections and microbial exposure are known to strongly affect the balance of the immune system in early life and among these CMV infection seems to be one of the strongest factors influencing the dynamics of the immune system in children [17]. Even though our study has a limitation of lacking information on confounding factors that affect microbial exposure in the perinatal period, e.g. information on the route of delivery and breastfeeding, a recently published study with the same DIPP cohort observed no association between breastfeeding and the emergence of islet autoimmunity or type 1 diabetes. This supports our interpretation that CMV infection is the main environmental factor behind the results observed here [18].

Our observation of the decelerating effect of early CMV infection on the progression rate of islet autoimmunity later in childhood could be caused by the strong, potentially tolerogenic immunomodulatory effect of CMV infection on T cells. The finding is in accordance with the hygiene hypothesis, which suggests that microbial exposures in early childhood stimulate immunoregulatory mechanisms that control allergic and autoimmune reactions. However, the 
exact immunological mechanisms behind this protective effect remain to be elucidated. Of note, we compared children with and without early CMV infection for the distribution of HLA genotypes associated with T1D but could not see any significant difference in the frequency of children positive for either DR3-DQ2, DR4-DQ8 or combination of both haplotypes between the groups (data not shown).

In conclusion, our current findings demonstrate a protective effect of CMV infection during early life on the development of clinical T1D among subjects at genetic risk for T1D. The results support the significance of the hygiene hypothesis for the pathogenesis of T1D and highlight the importance of early childhood infections for the maturation of healthy immune responses. 


\section{Acknowledgements}

We thank the personnel of the Immunogenetics Laboratory, University of Turku, especially Anne Suominen, Ritva Suominen, Piia Nurmi, Mia Karlsson and Terhi Laakso as well as the personnel and all participating families of the Finnish Diabetes Prediction and Prevention Study (DIPP).

\section{Funding}

The study has been supported by the Academy of Finland, Sigrid Jusélius Foundation, Juvenile (

Diabetes Research Foundation and Emil Aaltonen Foundation.

\section{8 \\ Duality of interest}

The authors declare that there is no duality of interest associated with this manuscript.

\section{Contribution statement}

$\mathrm{IE}, \mathrm{Jl}$ and $\mathrm{JL}$ researched data and wrote, reviewed and edited the manuscript. TV, MK, RV, JT, HH and TK contributed to acquisition, analysis and interpretation of data and critical revision of the manuscript. All listed authors approved the final version of the manuscript. IE takes responsibility for the integrity of the work as a whole. 


\section{References}

1. Rodriquez-Calvo T, Sabouri S, Anquetil F, von Herrath M.G. The viral paradigm in type 1 diabetes: Who are the main suspects? Autoimmun Rev. 2016; 15:964-969

2. Cannon MJ, Schmid DS, Hyde TB. Review of cytomegalovirus seroprevalence and demographic characteristics associated with infection. Rev Med Virol. 2010; 20:202-213

3. Alanen A, Kahala K, Vahlberg T, Koskela P, Vainionpää R. Seroprevalence, incidence of prenatal infections and reliability of maternal history of varicella zoster virus, cytomegalovirus, herpes simplex virus and parvovirus B16 in South-Western Finland. BJOG. 2005; 112:50-56

4. Aarnisalo J, llonen J, Vainionpää R, Volanen I, Kaitosaari T, Simell O. Development of antibodies against cytomegalovirus, Varicella-Zoster virus and Herpes simplex 1 virus in Finland during the first eight years of life: a prospective study. Scand J of Infect Dis. 2003; 35:750-753

5. Aarnisalo J, Veijola R, Vainionpää R, Simell O, Knip M, llonen J. Cytomegalovirus infection in early infancy: risk of induction and progression of autoimmunity associated with type 1 diabetes. Diabetologia 2008; 51:769-772

6. Ilonen J, Hammais A, Laine A-P, et al. Patterns of $\beta$-cell autoantibody appearance and genetic associations during the first years of life. Diabetes 2013; 62:3636-3640

7. Siljander HT, Simell S, Hekkala A et al. Predictive characteristics of diabetes-associated autoantibodies among children with HLA-conferred disease susceptibility in the general population. Diabetes 2009; 58:2835-2842

8. Ziegler T, Meurman O, Nikoskelainen J, Arstila P. Laboratory diagnosis of herpes virus infections in immunocompromised hosts. Ann Ist Super Sanita. 1987; 23:747-752

9. van der Werf N, Kroese F, Rozing J, Hillebrands J-L. Viral infections as potential triggers of type 1 diabetes. Diabetes Metab Res Rev. 2007; 23:169-183 
10. Op de Beeck A, Eizirik D.L. Viral infections in type 1 diabetes mellitus - why the $\beta$ cells. Nat Rev Endocrinol. 2016; 12:263-273

11. Ziegler AG, Bonifacio E; BABYDIAB-BABYDIET Study Group. Age-related islet autoantibody incidence in offspring of patients with type 1 diabetes. Diabetologia 2012; 55:1937-1943

12. Parikka V, Näntö-Salonen K, Saarinen M, et al. Early seroconversion and rapidly increasing autoantibody concentrations predict prepubertal manifestation of type 1 diabetes in children at genetic risk. Diabetologia 2012; 55:1926-1936

13. Plot L, Amital H, Barzilai O, Ram M, Nicola B, Shoenfeld Y. Infections may have a protective role in the pathogenesis of celiac disease. Ann. N.Y. Acad. Sci. 2009; 1173:670-674

14. Jansen M.A.E, van den Heuve D, van der Swet K.V.M., et al. Herpesvirus Infections and Transglutaminase type 2 Antibody Positivity in Childhood: The Generation R Study. J Pediatr Gastroenterol Nutr. 2016; 63:423-430

15. Khan N, Shariff N, Cobbold M, et al. Cytomegalovirus seropositivity drives the CD8 T cell repertoire toward greater clonality in healthy elderly individuals. J Immunol. 2002; 169:19841992

16. Almanzar G, Schwaiger S, Jenewein B, et al. Long-term cytomegalovirus infection leads to significant changes in the composition of the CD8+ T-cell repertoire, which may be the basis for imbalance in the cytokine production profile in elderly. J Virol. 2005; 79: 3675-3683

17. van den Heuvel D, Jansen $M$, Nasserinejad K, et al. Effects of nongetic factors on immune cell dynamics in early childhood: The generation R study. J Allergy Clin Immunol. 2017; 139:19231934

18. Hakola L, Takkinen H-M, Niinistö S, et al. Infant feeding in relation to the risk of advanced islet autoimmunity and type 1 diabetes in children with increased genetic susceptibility: a cohort study. Am J Epidemiol. 2018; 187:34-44 
Table 2. Characteristics of progression to islet autoimmunity and clinical type 1 diabetes

\begin{tabular}{|c|c|c|c|}
\hline Variable & All aab+ children & Non-progressor & Progressor \\
\hline Mean age (y) at sampling (+/- SD) & $1.47(1.22-1.72)$ & $1.50(1.28-1.72)$ & $1.46(1.19-1.73)$ \\
\hline $\begin{array}{l}\text { Mean age (y) at first double positive aab } \\
\text { time (range) }\end{array}$ & $3.28(0.34-13.99)$ & $4.72(0.58-13.99)$ & $2.48(0.34-10.60)$ \\
\hline & All children & CMV- & $\mathrm{CMV}+$ \\
\hline $\begin{array}{l}\text { Mean age (y) at first double positive aab } \\
\text { time (range) }\end{array}$ & $3.28(0.34-13.99)$ & $3.20(0.34-13.99)$ & $3.55(0.50-12.58)$ \\
\hline Mean age $(y)$ at onset of diabetes (range) & $6.55(0.82-14.88)$ & $6.46(0.82-14.56)$ & $6.91(1.03-14.88)$ \\
\hline $\begin{array}{l}\text { Mean time (y) of progression to diabetes } \\
\text { (range) }\end{array}$ & $4.07(0.0-12.24)$ & $4.06(0.0-11.78)$ & $4.37(0.2-12.24)$ \\
\hline
\end{tabular}




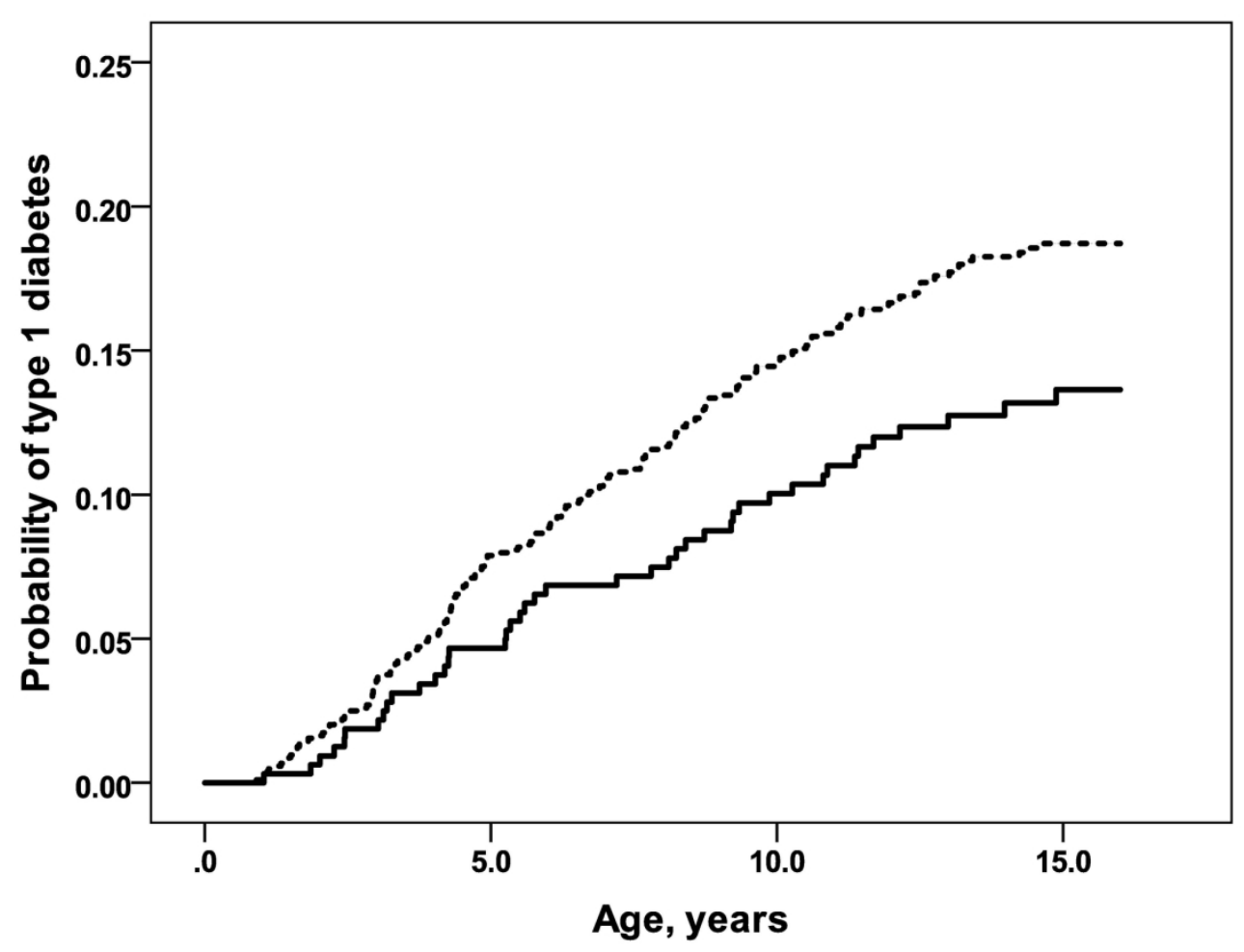

Figure 1. Progression to clinical type 1 diabetes among children with early cytomegalovirus infection (line) compared to children without the infection (dashed), $p=0.035$ log-rank test. Follow-up starting from birth. 


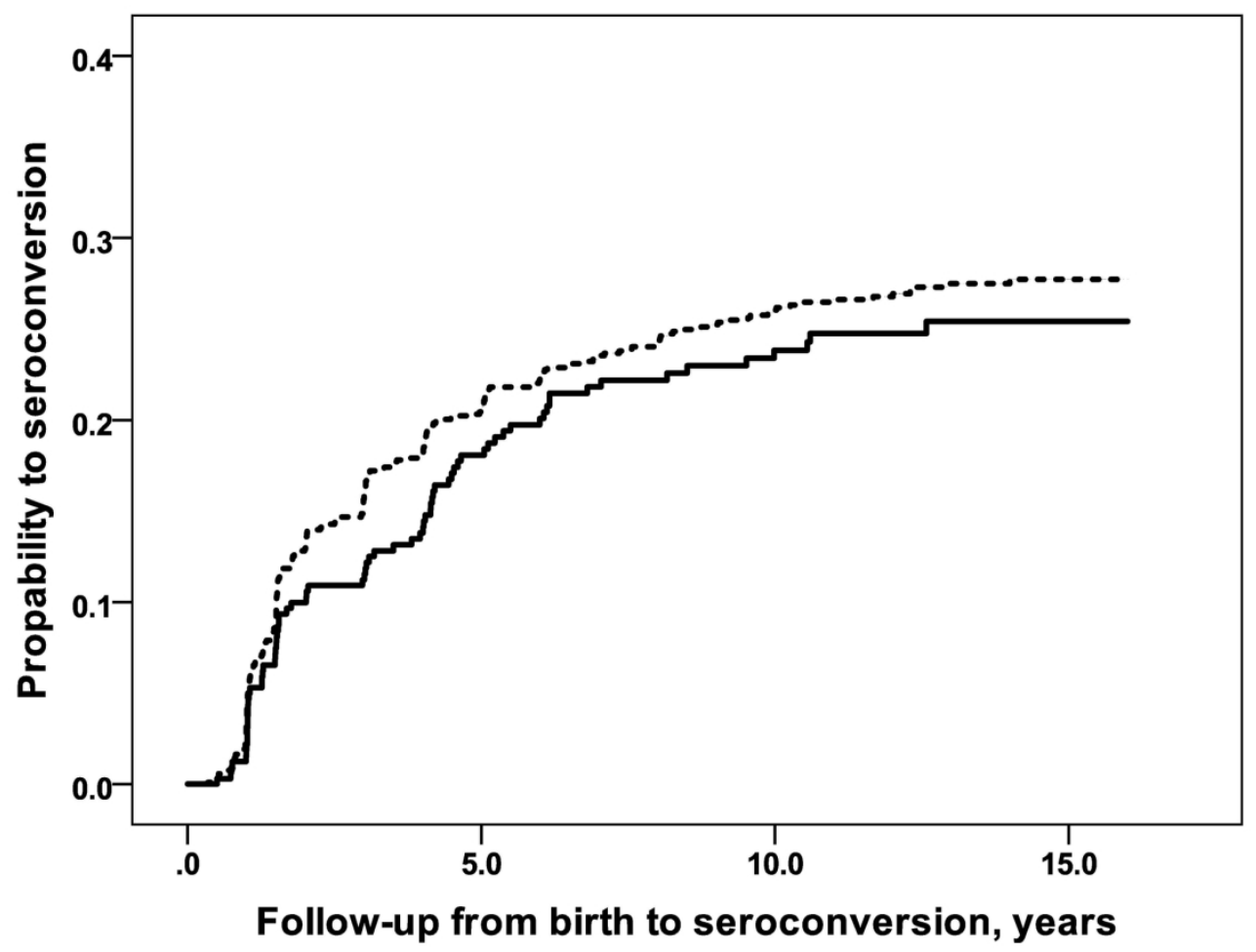

Figure 2. Seroconversion for islet autoantibodies among the children with early cytomegalovirus infection (line) compared to children without the infection (dashed), $p=0.357$. Follow-up starting from birth. 


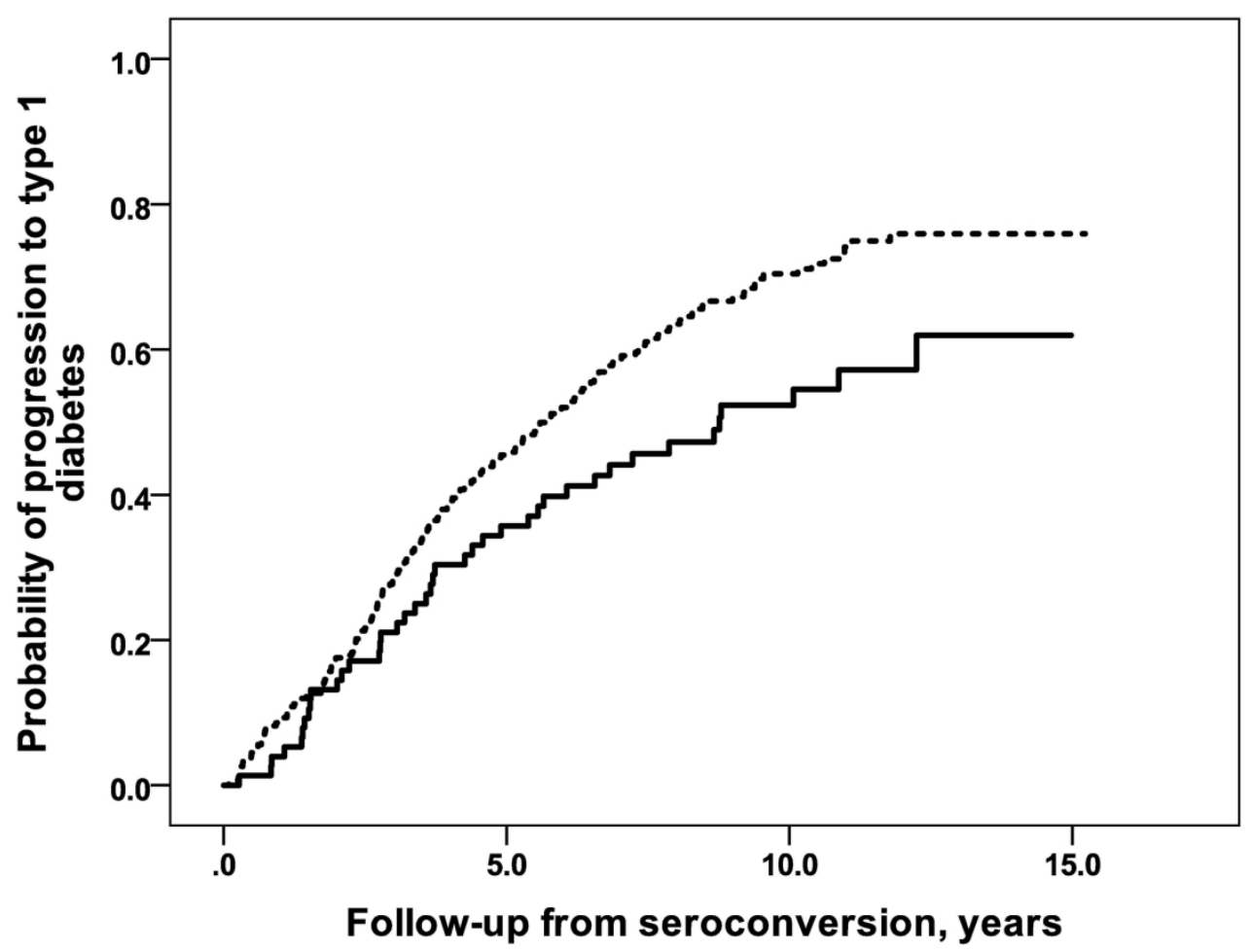

Figure 3. Progression to clinical type 1 diabetes after seroconversion to islet autoantibodies in children with CMV-specific IgG antibodies (line) compared to those without CMV antibodies (dashed), $p=0.015$. 